\title{
Räumliche und zeitliche Auflösung der Wiederherstellungsdynamik der Photorezeptoren nach Behandlung mit Voretigen Neparvovec
}

\author{
Krunoslav Stingl ${ }^{\text {a, b, c }}$ Melanie Kempf ${ }^{a}$, $\quad$ Karl U. Bartz-Schmidt ${ }^{a}$ Spyridon Dimopoulos ${ }^{a}$ \\ Felix Reichel $^{a}$ Ronja Jung ${ }^{a}$ Carina Kelbsch ${ }^{a, c}$ Susanne Kohl ${ }^{d}$ Friederike Charlotte Körtum $^{a}$ \\ Fadi Nasser $^{\mathrm{e}}$ Tobias Peters $^{c, f}$ Barbara Wilhelm ${ }^{c, f}$ Bernd Wissinger ${ }^{d}$ Fabian Wozar ${ }^{a}$ \\ Eberhart Zrenner ${ }^{b, e, g}$ M. Dominik Fischer ${ }^{a, e, h, i} \quad K^{2}$ atarina Stingl ${ }^{a, b}$ \\ aUniversitäts-Augenklinik, Department für Augenheilkunde, Universität Tübingen, Tübingen, Deutschland; ${ }^{b}$ Zentrum für seltene \\ Augenerkrankungen, Universität Tübingen, Tübingen, Deutschland; ${ }^{C}$ Forschungsgruppe Pupillenforschung an der Universitäts- \\ Augenklinik, Department für Augenheilkunde, Universität Tübingen, Tübingen, Deutschland; dMolekulargenetisches Labor, \\ Forschungsinstitut für Augenheilkunde, Department für Augenheilkunde, Universität Tübingen, Tübingen, Deutschland; \\ eForschungsinstitut für Augenheilkunde, Department für Augenheilkunde, Universität Tübingen, Tübingen, Deutschland; fSTZ eyetrial \\ am Department für Augenheilkunde, Universität Tübingen, Tübingen, Deutschland; 9 Werner Reichardt Centrum für Integrative \\ Neurowissenschaften (CIN), Universität Tübingen, Tübingen, Deutschland; h Oxford Eye Hospital, Oxford University Hospitals NHS \\ Foundation Trust, Oxford, Oxfordshire, Vereinigtes Königreich; 'Nuffield Laboratory of Ophthalmology, Department of Clinical \\ Neuroscience, University of Oxford, Oxford, Vereinigtes Königreich
}

\begin{abstract}
Hintergrund: Voretigen Neparvovec ist ein gentherapeutischer Wirkstoff zur Behandlung von Netzhautdystrophien, die durch biallelische RPE65-Mutationen verursacht werden. In der vorliegenden Studie berichten wir über eine neuartige und objektive Beurteilung der retinotopen Wiederherstellung der Photorezeptoren.

Methoden: Sieben (7) Augen von 5 Patienten (im Alter von 14, 21, 23, 24 und 36 Jahren, davon 1 Mann und 4 Frauen) mit biallelischen RPE65-Mutationen wurden mit Voretigen Neparvovec behandelt. Die klinischen Untersuchungen beinhalteten Visusbestimmungen, einen dunkeladaptierten Vollfeld-Lichtempfindlichkeits-Schwellenwert-Test (full-field stimulus threshold, FST), eine dunkeladaptierte chromatische Perimetrie (dark-adapted chromatic perimeter, DAC) mit einem 30-Grad-Raster und eine skotopische und photopische chromatische Pupillenkampimetrie (CPC) mit einem 30-Grad-Raster. Alle Untersuchungen und die Spectral-Domainoptische Kohärenztomographie wurden bei Baseline sowie nach 1 Monat und nach 3 Monaten durchgeführt.
\end{abstract}

M. Dominik Fischer und Katarina Stingl waren zu gleichen Teilen an der Erstellung dieser Arbeit beteiligt.
Ergebnisse: Außer bei dem ältesten Patienten war bei allen Patienten nach einem Monat eine messbare Verbesserung der Stäbchenfunktion gemäß FST, DAC oder skotopischer CPC nachweisbar. Der Visus verbesserte sich leicht oder blieb bei allen Augen stabil. Bei drei Augen zeigte sich eine Verbesserung der Zapfenfunktion in der photopischen CPC. Der Anstieg der dunkeladaptierten Lichtempfindlichkeitsschwelle beim FST-Test (blau) und den DAC-Reizen (cyan) korrelierte im Durchschnitt eng mit dem Alter $\left(R^{2}>0,7\right)$. Die Verbesserung der Pupillenreaktion in der skotopischen CPC korrelierte mit dem Ausgangswert des lokalen Netzhautvolumens $\left(R^{2}=0,5\right)$.

Schlussfolgerungen: Die vorgestellten Protokolle ermöglichen eine Beurteilung der individuellen räumlichen und zeitlichen Effekte der Gentherapie. Darüber hinaus untersuchten wir Parameter, die mit dem Therapieerfolg korrelierten. Die CPC und DAC, die komplementäre Aspekte der Netzhautfunktion messen, bieten eine neue und schnelle Möglichkeit, funktionelle Veränderungen in den retinotopen Karten der Stäbchen- und Zapfenfunktion zu beurteilen.

(c) Autor(en) (oder ihr(e) Arbeitgeber) 2021 


\section{Einleitung}

Voretigen Neparvovec ist eine zugelassene retinale Gentherapie zur Behandlung von Netzhautdystrophien, die durch biallelische RPE65-Mutationen verursacht werden. RPE65 wird in retinalen Epithelzellen exprimiert und kodiert für eine Retinoid-Isomerohydrolase, die Bestandteil des Sehzyklus zur Wiedergewinnung des Chromophors 11-cis-Retinal ist $[1,2]$. Netzhautdystrophien, die durch biallelische RPE65-Mutationen verursacht werden, manifestieren sich phänotypisch in der Regel durch eine früh einsetzende Netzhautdegeneration (early onset retinal degeneration, EORD) oder eine Lebersche kongenitale Amaurose Typ 2 (Leber congenital amaurosis type 2, LCA2) [3-5].

Die ersten erfolgreichen Ansätze zur Behandlung von LCA2 durch Genersatztherapie wurden 2008 vorgestellt $[6,7]$. Darin wurden Pilotergebnisse zur Sicherheit und Wirksamkeit der subretinalen Verabreichung von rekombinantem adeno-assoziiertem Virus, das ein RPE65-Transgen trägt, beschrieben [8, 9]. Es folgten verschiedene Veröffentlichungen, in denen über eine Verbesserung der Photorezeptorfunktion in Follow-up-Studien von bis zu 3 Jahren berichtet wurde [10-13]. Die 2017 veröffentlichten Ergebnisse einer klinischen Phase-3-Studie zeigten eine verbesserte Leistung im Multiluminanz-Mobilitätstest und bei der retinalen Empfindlichkeit gemäß Vollfeld-LichtempfindlichkeitsSchwellenwert-Test (full-field stimulus threshold, FST) bei $31 \mathrm{~Pa}-$ tienten ein Jahr nach der Intervention [14]. Die Nebenwirkungen im Zusammenhang mit der subretinalen Verabreichung des gentherapeutischen Wirkstoffs waren mit denen der Vitrektomie vergleichbar [14]. Im Jahr 2017 erteilte die US-amerikanische Behörde für Lebensmittel- und Arzneimittelsicherheit (Food and Drug Administration, FDA) die Zulassung für die kommerzielle Nutzung in den USA und in Europa erfolgte die Zulassung 2018 durch die Europäische Arzneimittelagentur (European Medicines Agency, EMA). Eine ausführliche Übersicht über die Sicherheit und Wirksamkeit vor der Zulassung wurde von Pierce und Bennett vorgelegt [15].
In den früheren Studien hatten sich nach der Therapie hauptsächlich die stäbchenvermittelten Messwerte verbessert, wie die FSTWerte sowie die Ganzfeldpupillographie und Mobilitätstests zeigten $[7,9,14]$. Nur wenige Veröffentlichungen berichteten über Verbesserungen der zapfenvermittelten Sehfunktionen [7, 10], während andere Publikationen keinerlei Verbesserungen der Zapfenfunktion dokumentierten [16, 17].

Mit der vorliegenden Studie sollte die longitudinale retinotope Veränderung der Stäbchen- und Zapfen-Photorezeptorfunktion mit neuartigen Methoden untersucht werden. Darüber hinaus wollten wir herausfinden, ob es Faktoren gibt, mit deren Hilfe sich der Behandlungseffekt vorhersagen lässt. Neben den klassischen Funktionsuntersuchungen wie FST und Visusbestimmung kamen dabei die dunkeladaptierte chromatische Perimetrie (DAC) mit einem neuen Protokoll und die chromatische Pupillenkampimetrie (CPC) zur Anwendung [18]. Diese neuartigen Protokolle können als klinische Instrumente dienen, die speziell auf die Beurteilung der Reaktion von Stäbchen und Zapfen nach der Behandlung von Erkrankungen der äußeren Netzhaut zugeschnitten sind.

Beide Methoden, die skotopische CPC und das gekürzte DACProtokoll, weisen eine hohe Zuverlässigkeit auf und ergänzen einander in der Fähigkeit zur Beurteilung verschiedener Aspekte der Stäbchenwiederherstellung [18-22].

\section{Material und Methoden}

\section{Patienten}

Nach Kostenübernahmezusage durch die jeweilige gesetzliche Krankenversicherung wurden 7 Augen von 5 EORD-Patienten (im Alter von 14, 21, 23, 24 und 36 Jahren, davon 1 Mann und 4 Frauen) mit Voretigen Neparvovec behandelt, das nach erfolgter Vitrektomie subretinal verabreicht wurde. Die Patientinnen P1 und P2 waren Schwestern, ebenso wie die Patientinnen P3 und P4. Die genetische Analyse ergab bei Patientin P1 und P2 eine homozygote c.1451G>T/p.(Gly484Val)-Mutation im RPE65-Gen

Tab. 1. Klinische Merkmale der Augen bei Baseline

\begin{tabular}{|c|c|c|c|c|}
\hline & Geschlecht & $\begin{array}{l}\text { BCVA } \\
\text { (dezimal) }\end{array}$ & $\begin{array}{l}\text { FST blau } \\
\left(0 \mathrm{~dB}=0,01 \mathrm{~cd} / \mathrm{m}^{2}\right)\end{array}$ & $\begin{array}{l}\text { FST rot } \\
\left(0 \mathrm{~dB}=0,01 \mathrm{~cd} / \mathrm{m}^{2}\right)\end{array}$ \\
\hline P1 RA & Weiblich & 0,125 & $-0,83 \mathrm{~dB}$ & $1,5 \mathrm{~dB}$ \\
\hline P1 LA & Weiblich & 0,16 & $-9,71 \mathrm{~dB}$ & $-1,93 \mathrm{~dB}$ \\
\hline P2 LA & Weiblich & 0,025 & $0,22 \mathrm{~dB}$ & $3,7 \mathrm{~dB}$ \\
\hline P3 LA & Weiblich & 0,06 & $-0,72 \mathrm{~dB}$ & $2,73 \mathrm{~dB}$ \\
\hline P4 RA & Weiblich & $\mathrm{FZ}$ & $-4,15 \mathrm{~dB}$ & $0,85 \mathrm{~dB}$ \\
\hline P4 LA & Weiblich & $\mathrm{FZ}$ & $-0,91 \mathrm{~dB}$ & $3,18 \mathrm{~dB}$ \\
\hline P8 LA & Männlich & 0,2 & $-7,6 \mathrm{~dB}$ & $-4,21 \mathrm{~dB}$ \\
\hline
\end{tabular}

Es wurden 7 Augen von 5 Patienten mit biallelischen RPE65-Mutationen mit Voretigen Neparvovec behandelt. Alle Patienten wiesen vor der Behandlung keine messbare Stäbchenfunktion und eine verminderte Sehschärfe auf. BCVA: best corrected visual acuity (bestkorrigierter Visus); FZ: Fingerzählen; FST: full-field stimulus threshold (Vollfeld-Lichtempfindlichkeits-Schwellenwert-Test); LA: linkes Auge; RA: rechtes Auge. 
und bei Patientin P3 und P4 eine homozygote c.1102T $>C / p$. (Tyr368His)-Mutation im RPE65-Gen. Patient P8 war compoundheterozygot für die Mutationen c.208T $>\mathrm{G} / \mathrm{p}$.(Phe70Val) und c.246-11A>G;p.(?). In allen Fällen erfolgte eine Segregationsanalyse bei beiden Elternteilen der Patienten.

Alle Patienten, über die in der vorliegenden Arbeit berichtet wird, erhielten Voretigen Neparvovec am Zentrum für Augenheilkunde der Universität Tübingen und stimmten der Auswertung ihrer klinischen Daten im Rahmen dieses Projekts zu. Abgesehen von einer vorherigen Behandlung mit Voretigen Neparvovec am Zentrum für Augenheilkunde der Universität Tübingen galten für die Aufnahme in diese Analyse keine Einschlusskriterien. Die Eignungskriterien für den Erhalt dieser Behandlung entsprachen den allgemeinen Empfehlungen und denen der Deutschen Ophthalmologischen Gesellschaft [23]. Die Analyse der klinischen Daten erfolgte gemäß den Grundsätzen der Deklaration von Helsinki. Von allen Patienten wurde eine schriftliche Einwilligungserklärung eingeholt.

Die präoperativen klinischen Befunde der Patienten sind in Tabelle 1 aufgeführt. Sämtliche dunkeladaptierten Funktionstests (DAC, FST, skotopische CPC) erbrachten bei allen sieben Augen die Bestätigung, dass vor der Operation keine messbare Stäbchenfunktion bestand. Die photopische CPC und der bestkorrigierte Visus (best corrected visual acuity, BCVA) ergaben ein unterschiedliches Ausmaß an messbarer Zapfenfunktion vor der Operation.

\section{Chirurgische Verabreichung}

Es erfolgte eine Standard-Pars-plana-Vitrektomie mit 23 G-Trokar und die Ablösung der posterioren Glaskörpermembran wurde durch Injektion von Triamcinolon bestätigt, wenn der Operateur dies für erforderlich hielt. Mit einer 41-G-Kanüle wurde die Retinotomie entlang des oberen Gefäßbogens angelegt und mithilfe eines über Fußschalter bedienbaren Injektionssystems wurden $0,3 \mathrm{ml}$ Vektorlösung in den subretinalen Raum unter Ausrichtung auf die Makula injiziert. Der Eingriff erfolgte gemäß den Empfehlungen der Deutschen Ophthalmologischen Gesellschaft [23].

\section{Klinische Untersuchungen}

Die in dieser Arbeit vorgestellten klinischen Untersuchungen umfassten Messungen des bestkorrigierten Visus mit ETDRS-Tafeln und dunkeladaptierte skotopische Vollfeld-Lichtempfindlichkeits-Schwellenwert-Tests mit blauem und rotem Licht (FST, Diagnosys LLC, Cambridge, UK), wobei $0 \mathrm{~dB}$ auf $0,01 \mathrm{~cd} / \mathrm{m}^{2}$ festgelegt war. Ferner erfolgte eine Bestimmung der dunkeladaptierten Netzhautempfindlichkeit mit dem Medmont Dark Adapted Chromatic (DAC) Perimeter (Medmont Pty Ltd International, Victoria, Australien) unter Verwendung eines neuartigen, kürzeren Protokolls mit 36 Testpunkten in den zentralen 30 Grad [18, 19]. Für den cyanfarbenen Lichtreiz mit $505 \mathrm{~nm}$ Wellenlänge betrug der dynamische Bereich $0 \mathrm{~dB}$ bis $-75 \mathrm{~dB}(0 \mathrm{~dB}$ entspricht 17,6 $\mathrm{cd} / \mathrm{m} 2)$ und für den roten Lichtreiz mit $625 \mathrm{~nm}$ Wellenlänge $0 \mathrm{~dB}$ bis - $50 \mathrm{~dB}$. Folgende Daten werden in der vorliegenden Arbeit be- richtet: 1) Sensitivitätskarten des gesamten 30 Grad-Bereichs, 2) durchschnittliche Empfindlichkeit der 15-Grad-Makularegion und 3) gemittelte Empfindlichkeit in den vier Quadranten der mittels optischer Kohärenztomographie (optical coherence tomography, OCT) durchgeführten Netzhautvolumenanalyse (Abb. 1 und 2).

Für eine objektive Beurteilung der lokalen Stäbchen- und Zapfenfunktion im zentralen 30-Grad-Gesichtsfeld wurde bei allen Visiten eine CPC mit einem spezifischen skotopischen Protokoll (die Stäbchen begünstigendes Protokoll mit blauen Lichtreizen) und photopischen Protokoll (die Zapfen begünstigendes Protokoll mit roten Lichtreizen) durchgeführt [18, 22, 24]. Die Präsentation der Lichtreize erfolgte auf einem OLED-Breitbildmonitor (organische Leuchtdiode) innerhalb der zentralen Exzentrizität von $30 \mathrm{Grad}$ mit einem Blickverfolgungsalgorithmus für eine korrekte Retinotopie; eine Infrarotkamera zeichnete währenddessen kontinuierlich den Pupillendurchmesser auf. Für das photopische Protokoll wurden rote Lichtreize vor einem schwachen blauen Hintergrund präsentiert (Radius der Lichtreize: 3 Grad; Dauer der Lichtreize: $1 \mathrm{~s}$; Intensität der Lichtreize: $60 \mathrm{~cd} / \mathrm{m}^{2}$; Wellenlänge der Lichtreize: $620 \mathrm{~nm} \pm 30 \mathrm{~nm}$ ). Für das skotopische Protokoll wurden nach 20-minütiger Dunkeladaptation schwache blaue Lichtreize präsentiert (Radius der Lichtreize: 5 Grad; Dauer der Lichtreize: $100 \mathrm{~ms}$; Intensität der Lichtreize: $0,01 \mathrm{~cd} / \mathrm{m}^{2}$; Wellenlänge der Lichtreize: $460 \mathrm{~nm} \pm 30 \mathrm{~nm}$ ). Für jede Reizlokalisation wurde die relative maximale Amplitude der Pupillenverengung (relMCA; prozentualer Anteil der Pupillenverengung nach der Reizpräsentation gegenüber dem Ausgangswert des $\mathrm{Pu}$ pillendurchmessers) berechnet [25]. Folgende Daten werden in der vorliegenden Arbeit berichtet: 1) Karte der relMCA des gesamten 30-Grad-Bereichs, 2) durchschnittliche relMCA von den untersuchten Punkten der makulären 15-Grad-Region und 3) durchschnittliche relMCA innerhalb der vier Quadranten der OCT-Netzhautvolumenanalyse (Abb. 1 und 2).

Die Aufnahme der Spektral-Domänen-OCT (SD-OCT)-Bilder erfolgte mit dem Spectralis HRA+OCT-System (Heidelberg Engineering $\mathrm{GmbH}$, Heidelberg). Bei Baseline und bei den Followup-Visiten wurden horizontale und vertikale Einzel-B-Scans der Fovea aufgenommen und bei ausreichend guter Fixation wurden zusätzliche Volumenscans $\left(15^{\circ} \times 15^{\circ}\right)$ erstellt. Die lokalen Netzhautvolumina wurden verwendet, um die Korrelation mit den funktionellen Messwerten zu ermitteln. Die Berechnung des lokalen Netzhautvolumens erfolgte mit dem 1-, 2,22- und 3,45-mmRaster, das auf die Fovearegion zentriert war, wobei vier Quadranten ausgewertet wurden: superior, temporal, inferior und nasal jeweils $1 \mathrm{~mm}$ bis $3,45 \mathrm{~mm}$ (entsprechend etwa 3 bis $12 \mathrm{Grad}$, siehe Abb. 1 und 2).

\section{Statistische Methoden}

Der Einfluss des Alters auf die Empfindlichkeitsänderung (DAC und FST) nach der Behandlung und die Amplitude der Pupillenreaktion 1 und 3 Monate nach der Intervention wurde mittels Regressionsanalyse untersucht. Nur für vier Augen (P1 RA, P1 LA, P3 LA und P8 LA) lagen OCT-Daten zum Netzhautvolumen vor der Be-
156

Kompass Ophthalmol 2021;7:154-163 DOI: $10.1159 / 000519131$ 


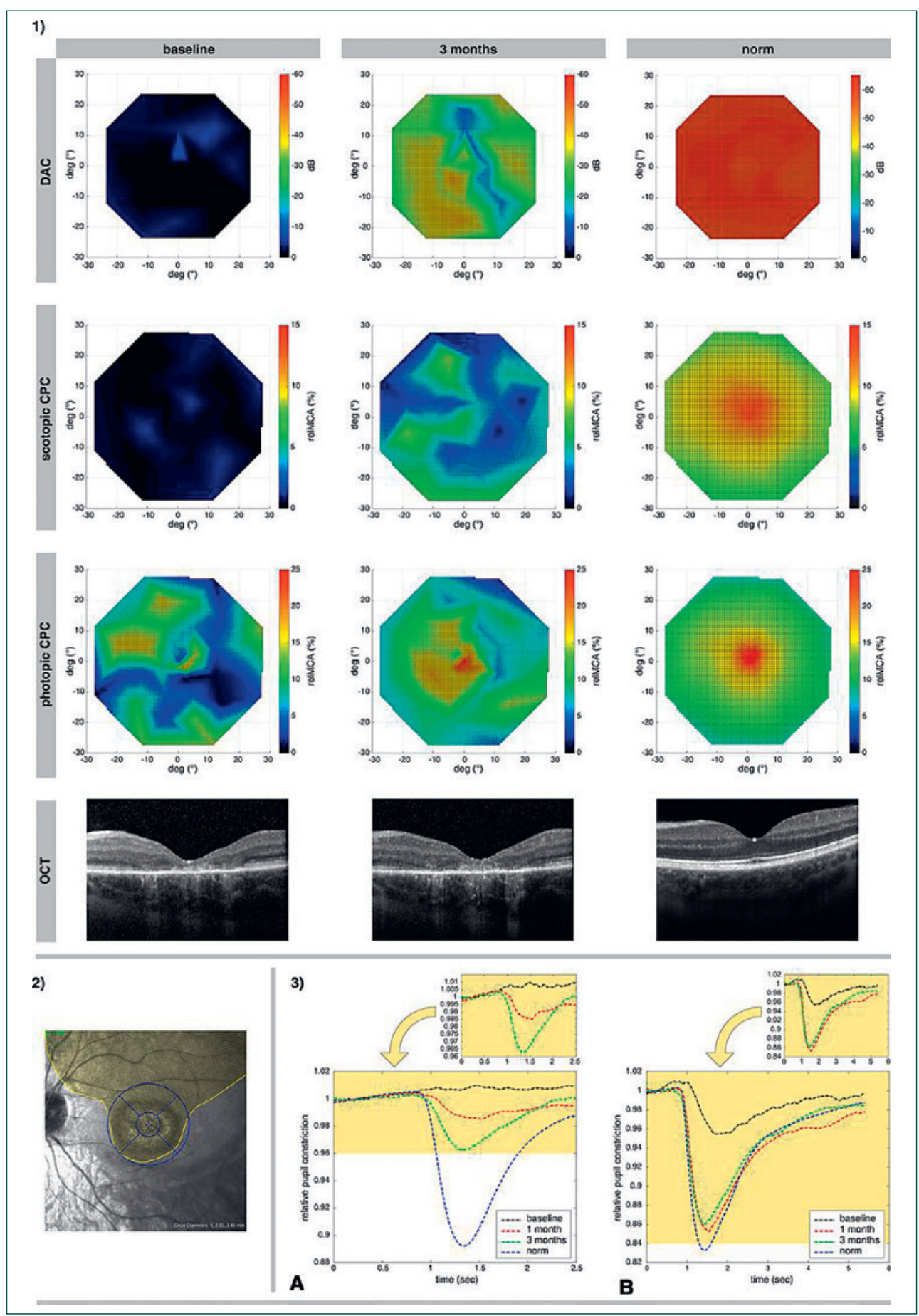

Abb. 1. Darstellung der Behandlungseffekte bei Patientin P3, der jüngsten Patientin, die gut auf die Therapie ansprach. 1) Retinotopie der Wiederherstellung der Photorezeptoren, gemessen mittels DAC cyan (obere Zeile; der farbkodierte Balken stellt die Abnahme des Schwellenwerts gegenüber 0 dB dar), skotopischer CPC und photopischer CPC (zweite und dritte Zeile; der farbkodierte Balken stellt den relMCA-Wert in \% dar). Die untere Zeile zeigt die fovealen horizontalen Linien der OCT-Aufnahmen, die bei denselben Visiten gemacht wurden. Die Ergebnisse sind im Vergleich zu einer normativen Messung an gesunden Augen dargestellt (rechte Spalte). 2) Die Ausdehnung des bei der Verabreichung von Voretigen Neparvovec chirurgisch induzierten subretinalen Bläschens im linken Auge von Patientin P3 ist gelb umrandet, mit einer Überlagerung des OCT-Rasters, das für die Berechnungen der Netzhautvolumina verwendet wurde (innerer Kreis: ca. 3-Grad-Makularegion; äußerer Kreis: 12-Grad-Makularegion, weiter unterteilt in die vier Quadranten der OCT-Netzhautvolumenanalyse). 3) Gemittelte relative Pupillenreaktion im 15-Grad-Makulabereich von Patientin P3 für skotopische (A) und photopische (B) Lichtreize im Zeitverlauf. Bei Baseline (schwarze Linien) war keine messbare Reaktion der Pupille auf skotopische Reize und eine verminderte Pupillenreaktion auf photopische Lichtreize nachweisbar. Die nach einem Monat zu beobachtende Verbesserung (rote Linien) stieg nach drei Monaten (grüne Linien) bei der skotopischen Reaktion weiter an und blieb für die photopische Reaktion bei nahezu normalen Werten stabil. In den Diagrammen unten sind die Reaktionen auf skotopische und photopische Lichtreize in Relation zu den normativen Reaktionen gesunder Augen (blaue Linien) dargestellt. CPC: chromatic pupil campimetry (chromatische Pupillenkampimetrie); DAC: dark-adapted chromatic perimeter (dunkeladaptierte chromatische Perimetrie); OCT: optical coherence tomography (optische Kohärenztomographie); relMCA: relative maximal constriction amplitude (relative maximale Amplitude der Pupillenverengung). 


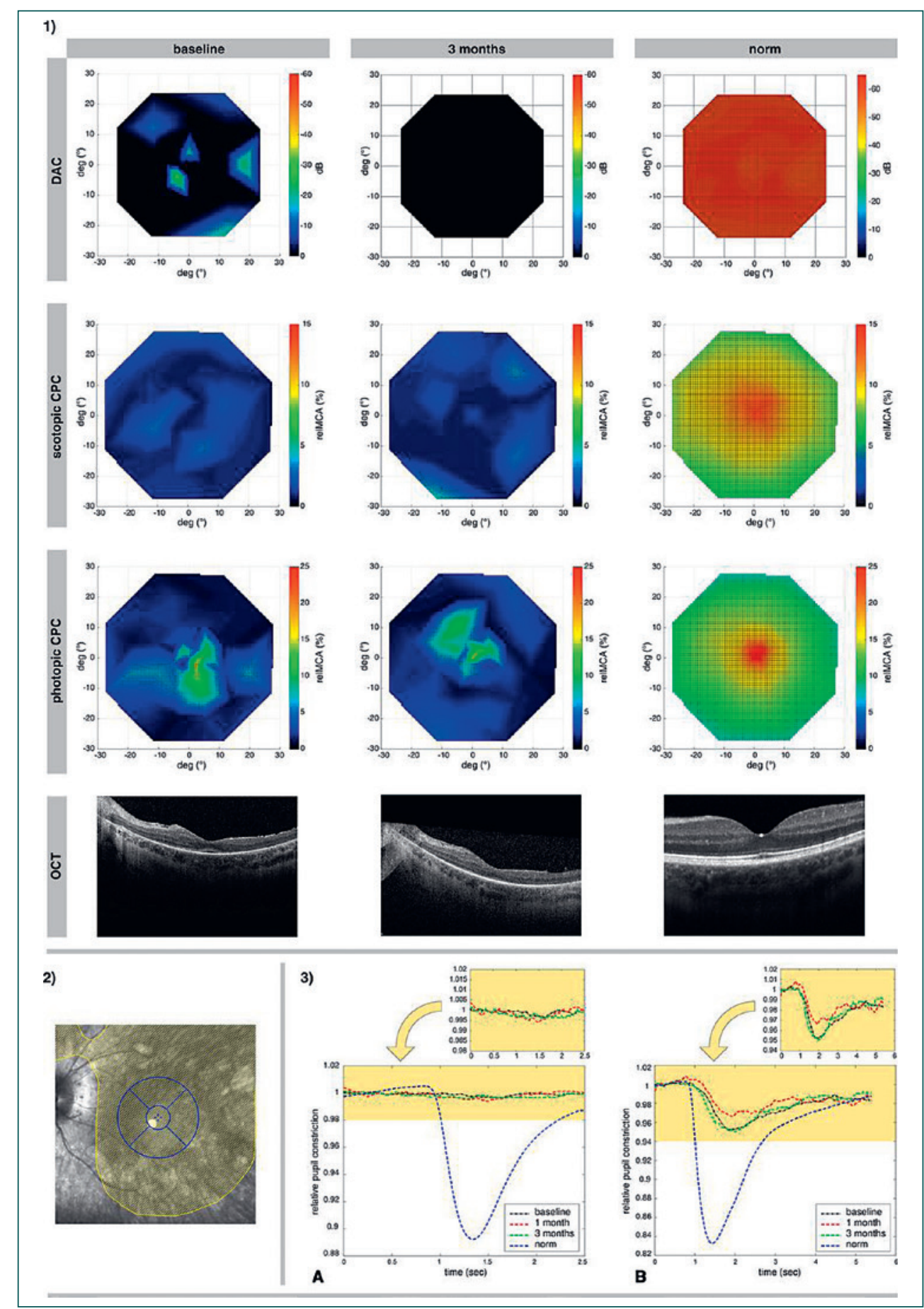

Abb. 2. Darstellung der Behandlungseffekte bei Patientin P4, der ältesten Patientin (Non-Responder). 1) Retinotopie der Wiederherstellung der Photorezeptoren, gemessen mittels DAC cyan (obere Zeile; der farbkodierte Balken stellt die Abnahme des Schwellenwerts gegenüber 0 dB dar), skotopischer CPC und photopischer CPC (zweite und dritte Zeile; der farbkodierte Balken stellt den relMCA-Wert in \% dar). Die vierte Zeile zeigt die fovealen horizontalen Linien der OCTAufnahmen, die bei denselben Visiten gemacht wurden. Die Ergebnisse sind im Vergleich zu einer normativen Messung an gesunden Augen dargestellt (rechte Spalte). 2) Das bei der Verabreichung von Voretigen Neparvovec chirurgisch induzierte subretinale Bläschen im linken Auge von Patientin P4 ist gelb umrandet, mit einer Überlagerung des OCT-Rasters, das für die Berechnungen der Netzhautvolumina verwendet wurde (innerer Kreis: ca. 3-Grad-Makularegion; äußerer Kreis: 12-Grad-Makularegion, weiter unterteilt in die vier Quadranten der OCT-Netzhautvolumenanalyse). 3) Gemittelte relative Pupillenreaktion im 15-Grad-Makulabereich von Patientin P3 für skotopische (A) und photopische (B) Lichtreize im Zeitverlauf. Bei Baseline (schwarze Linien) war keine messbare Reaktion der Pupille auf skotopische Reize und eine verminderte Pupillenreaktion auf photopische Lichtreize nachweisbar. Weder nach Monat 1 (rote Linien) noch nach Monat 3 (grüne Linien) waren Verbesserungen messbar. In den Diagrammen unten sind die Reaktionen auf skotopische und photopische Lichtreize in Relation zu den normativen Reaktionen gesunder Augen (blaue Linien) dargestellt. CPC: chromatic pupil campimetry (chromatische Pupillenkampimetrie); DAC: dark-adapted chromatic perimeter (dunkeladaptierte chromatische Perimetrie); OCT: optical coherence tomography (optische Kohärenztomographie); relMCA: relative maximal constriction amplitude (relative maximale Amplitude der Pupillenverengung). 
Table 2. Funktionswerte der behandelten Patientenaugen

\begin{tabular}{|c|c|c|c|c|c|c|c|c|c|}
\hline $\begin{array}{l}\text { ID } \\
\text { Auge }\end{array}$ & & $\begin{array}{l}\text { P1 } \\
\text { RA }\end{array}$ & $\begin{array}{l}\text { P1 } \\
\text { LA }\end{array}$ & $\begin{array}{l}\text { P2 } \\
\text { LA }\end{array}$ & $\begin{array}{l}\text { P3 } \\
\text { LA }\end{array}$ & $\begin{array}{l}\text { P4 } \\
\text { RA }\end{array}$ & $\begin{array}{l}\text { P4 } \\
\text { LA }\end{array}$ & $\begin{array}{l}\text { P8 } \\
\text { LA }\end{array}$ & Durchschnitt \\
\hline BCVA & $\begin{array}{l}\text { Baseline } \\
\text { Monat } 1 \\
\text { Monat } 3\end{array}$ & $\begin{array}{l}0,125 \\
0,125 \\
0,2\end{array}$ & $\begin{array}{l}0,16 \\
0,16 \\
0,1\end{array}$ & $\begin{array}{l}0,025 \\
0,025 \\
0,025\end{array}$ & $\begin{array}{l}0,06 \\
0,1 \\
0,125\end{array}$ & $\begin{array}{l}F Z \\
0,025 \\
0,02\end{array}$ & $\begin{array}{l}F Z \\
0,03 \\
0,02\end{array}$ & $\begin{array}{l}0,2 \\
0,2 \\
0,25\end{array}$ & \\
\hline FST blau (dB) & $\begin{array}{l}\text { Baseline } \\
\text { Monat } 1 \\
\text { Monat } 3\end{array}$ & $\begin{array}{l}-0,83 \\
-26,20 \\
-18,77\end{array}$ & $\begin{array}{l}-9,71 \\
-18,84 \\
-25,69\end{array}$ & $\begin{array}{c}0,22 \\
-11,11 \\
-6,29\end{array}$ & $\begin{array}{l}-0,72 \\
-44,00 \\
-29,10\end{array}$ & $\begin{array}{l}-4,15 \\
-2,06 \\
-6,07\end{array}$ & $\begin{array}{l}-0,91 \\
-5,55 \\
-6,83\end{array}$ & $\begin{array}{l}-7,60 \\
-34,73 \\
-23,44\end{array}$ & $\begin{array}{l}-3,39 \\
-20,36 \\
-16,60\end{array}$ \\
\hline FST rot $(\mathrm{dB})$ & $\begin{array}{l}\text { Baseline } \\
\text { Monat } 1 \\
\text { Monat } 3\end{array}$ & $\begin{array}{c}1,5 \\
-7,72 \\
-11,05\end{array}$ & $\begin{array}{l}-1,93 \\
-9,1 \\
-7,02\end{array}$ & $\begin{array}{c}3,7 \\
-0,48 \\
1,96\end{array}$ & $\begin{array}{c}0,85 \\
-18,68 \\
-9,56\end{array}$ & $\begin{array}{r}3,18 \\
4,85 \\
-0,23\end{array}$ & $\begin{array}{r}2,73 \\
-6,09 \\
1,58\end{array}$ & $\begin{array}{l}-4,21 \\
-10,88 \\
-6,49\end{array}$ & $\begin{array}{r}0,83 \\
-6,87 \\
-4,40\end{array}$ \\
\hline DAC blau Durchschnitt (dB) & $\begin{array}{l}\text { Baseline } \\
\text { Monat } 1 \\
\text { Monat } 3\end{array}$ & $\begin{array}{l}-1,10 \\
-18,50 \\
-21,90\end{array}$ & $\begin{array}{c}0,00 \\
-14,80 \\
-18,70\end{array}$ & $\begin{array}{r}0,00 \\
-0,60 \\
0,00\end{array}$ & $\begin{array}{l}-1,40 \\
-25,40 \\
-28,40\end{array}$ & $\begin{array}{l}0,00 \\
0,00 \\
0,00\end{array}$ & $\begin{array}{r}-2,70 \\
-0,50 \\
0,00\end{array}$ & $\begin{array}{l}-15,20 \\
-21,60 \\
-24,00\end{array}$ & $\begin{array}{l}-2,91 \\
-11,63 \\
-13,29\end{array}$ \\
\hline DAC rot Durchschnitt (dB) & $\begin{array}{l}\text { Baseline } \\
\text { Monat } 1 \\
\text { Monat } 3\end{array}$ & $\begin{array}{r}0,00 \\
-4,20 \\
-5,20\end{array}$ & $\begin{array}{l}-0,75 \\
-2,20 \\
-3,20\end{array}$ & $\begin{array}{l}0,00 \\
0,00 \\
0,00\end{array}$ & $\begin{array}{l}-3,20 \\
-14,10 \\
-12,90\end{array}$ & $\begin{array}{l}0,00 \\
0,00 \\
0,00\end{array}$ & $\begin{array}{l}0,00 \\
0,00 \\
0,00\end{array}$ & $\begin{array}{l}-3,10 \\
-6,30 \\
-5,80\end{array}$ & $\begin{array}{l}-1,01 \\
-3,83 \\
-3,87\end{array}$ \\
\hline $\begin{array}{l}\text { CPC Stäbchen Durchschnitt } \\
\text { (relMCA) }\end{array}$ & $\begin{array}{l}\text { Baseline } \\
\text { Monat } 1 \\
\text { Monat } 3\end{array}$ & $\begin{array}{l}0,00 \\
1,07 \\
2,00\end{array}$ & $\begin{array}{l}0,21 \\
1,18 \\
0,32\end{array}$ & $\begin{array}{l}0,06 \\
0,06 \\
0,00\end{array}$ & $\begin{array}{l}0,00 \\
2,58 \\
3,52\end{array}$ & $\begin{array}{l}0,41 \\
0,48 \\
0,35\end{array}$ & $\begin{array}{l}0,59 \\
0,71 \\
0,41\end{array}$ & $\begin{array}{l}0,89 \\
4,61 \\
4,07\end{array}$ & $\begin{array}{l}0,31 \\
1,53 \\
1,52\end{array}$ \\
\hline $\begin{array}{l}\text { CPC Zapfen Durchschnitt } \\
\text { (relMCA) }\end{array}$ & $\begin{array}{l}\text { Baseline } \\
\text { Monat } 1 \\
\text { Monat } 3\end{array}$ & $\begin{array}{l}5,40 \\
6,37 \\
4,20\end{array}$ & $\begin{array}{l}1,27 \\
4,62 \\
5,12\end{array}$ & $\begin{array}{l}0,37 \\
3,92 \\
0,90\end{array}$ & $\begin{array}{l}4,60 \\
14,75 \\
14,00\end{array}$ & $\begin{array}{l}4,30 \\
4,91 \\
4,41\end{array}$ & $\begin{array}{l}4,88 \\
3,34 \\
5,03\end{array}$ & $\begin{array}{l}8,03 \\
8,37 \\
5,54\end{array}$ & $\begin{array}{l}4,12 \\
6,61 \\
5,60\end{array}$ \\
\hline
\end{tabular}

Bei den aufgeführten Werten handelt es sich um die Funktionsmesswerte der behandelten Augen bei Baseline sowie 1 Monat und 3 Monate nach der Injektion. Der bestkorrigierte Visus (BCVA) ist in Dezimalwerten angegeben. Der FST ist in dB angegeben $\left(0 \mathrm{~dB}=0,01 \mathrm{~cd} / \mathrm{m}^{2}\right)$. Die DAC-Werte zeigen die durchschnittliche Empfindlichkeit in einem Bereich von 15 Grad um die Makula, was dem behandelten Areal entspricht $\left(0 \mathrm{~dB}=17,6 \mathrm{~cd} / \mathrm{m}^{2}\right)$. Die CPC-Werte zeigen die durchschnittliche relative maximale Verengungsamplitude (reIMCA) in einem Bereich von 15 Grad um die Makula, was dem behandelten Areal entspricht. BCVA: best corrected visual acuity (bestkorrigierter Visus); CPC: chromatic pupil campimetry (chromatische Pupillenkampimetrie); DAC: dark-adapted chromatic perimeter (dunkeladaptierte chromatische Perimetrie); FZ: Fingerzählen; FST: full-field stimulus threshold (Vollfeld-Lichtempfindlichkeits-Schwellenwert-Test); LA: linkes Auge; RA: rechtes Auge.

handlung vor. Für die (oben beschriebenen) Volumensegmente der Baseline-OCT wurden Regressionsanalysen mit den entsprechenden Werten der funktionellen Messungen (DAC und CPC) durchgeführt, was 16 Datenpunkte für die Regressionsanalyse ergab.

\section{Ergebnisse}

In Tabelle 2 sind alle funktionellen Messwerte bei Baseline sowie 1 Monat und 3 Monate nach der Behandlung mit Voretigen Neparvovec aufgeführt.

\section{Skotopische Messwerte nach der Behandlung}

Bis auf eine Patientin (P4, Abb. 2) zeigten alle Patienten eine Verbesserung der Stäbchenfunktion gemäß FST (blau), DAC (cyan) oder skotopischer CPC (Tabelle 2). Die Abnahme der dunkeladaptierten Lichtempfindlichkeitsschwelle betrug bei der jüngsten Patientin (P3) > $40 \mathrm{~dB}$ (FST und DAC) (Abb. 1). Der Anstieg der relMCA erreichte bei P3 an einigen behandelten Netzhautlokalisationen 7\% (Abb. 1 und 2). Bei Patientin P2 zeigte sich nach 1 Monat eine leichte Verbesserung der dunkeladaptierten Stäbchenempfindlichkeit gemäß FST (blau), die jedoch in der skotopischen CPC oder DAC nicht nachgewiesen wurde.
In Abbildung 1 sind die funktionellen Netzhautkarten von Patientin P3 (Responder) dargestellt, die eine deutliche Erholung der Stäbchen- und Zapfenfunktion 3 Monate nach der Behandlung erkennen lassen. Die zeitliche Dynamik der Pupillenreaktion aus den Bereichen der Netzhaut, die sich erholt hatten, weist normale Merkmale bezüglich Reaktionsbeginn und Reaktionsmaximum auf, was auf eine normale Einbeziehung der Stäbchen in das retinale Netzwerk hindeutet.

In Abbildung 2 sind die funktionellen Netzhautkarten von Patientin P4 (LA) dargestellt, die keine messbare Verbesserung der Zapfen- und Stäbchenfunktion zeigen.

\section{Photopische Messwerte nach der Behandlung}

Der Visus verbesserte sich leicht oder blieb bei allen Augen stabil. Bei Patient P8 kam es nach der Operation zu einer subjektiven Verschlechterung des bestkorrigierten Visus, die durch eine foveale Fibrose nach der Behandlung verursacht wurde. In drei Augen (P1 LA, P3 LA und P2 LA nur nach 1 Monat) war in der photopischen CPC eine Verbesserung der Zapfenfunktion zu beobachten. Abbildung 1 zeigt die Verbesserung bei Patientin P3, die über 3 Monate anhielt, und ein Beispiel für das Ansprechen auf die Therapie darstellt. 


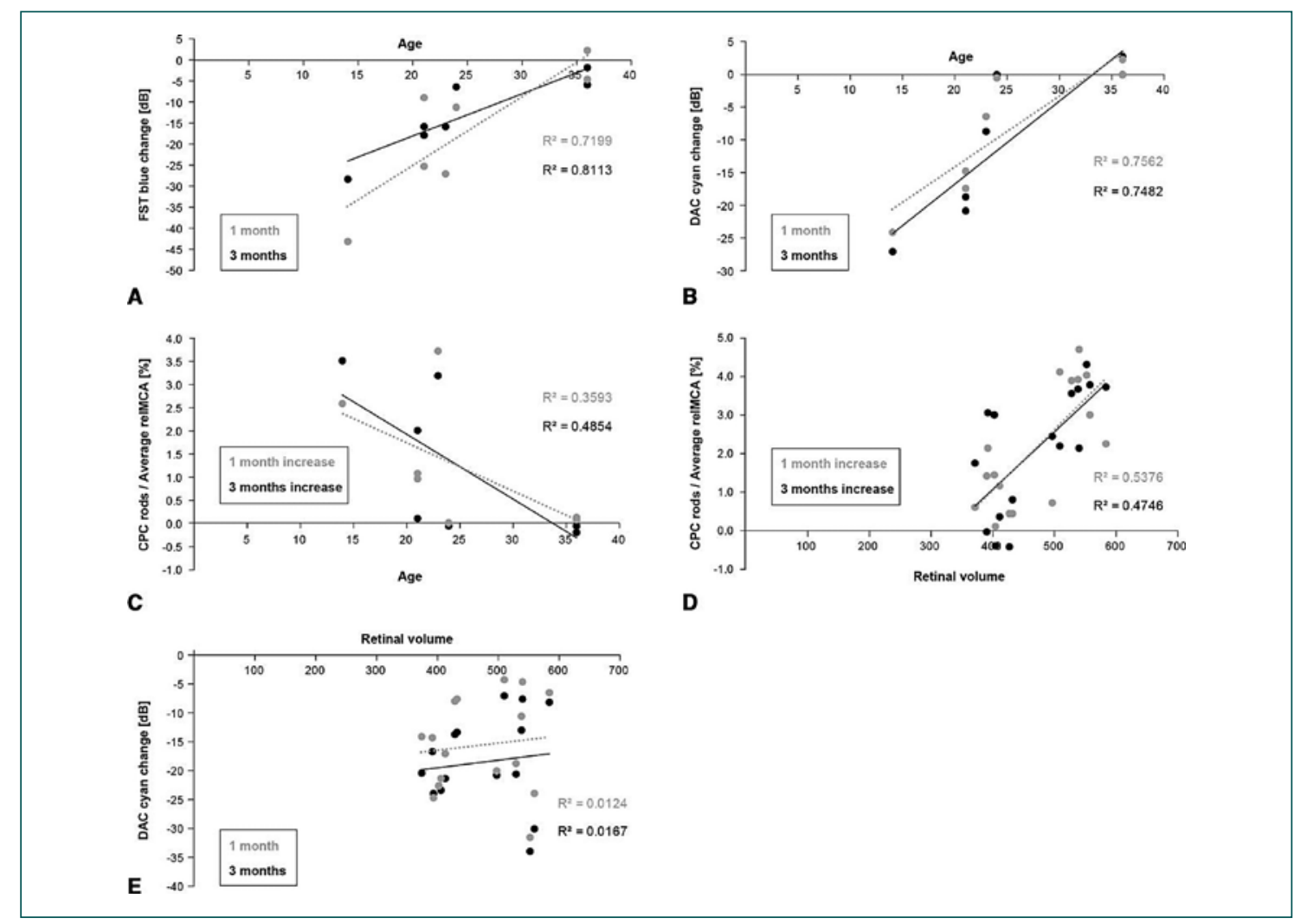

Abb. 3. Analyse der prädiktiven Faktoren. (A) Korrelation zwischen dem Alter und der Verbesserung des dunkeladaptierten Lichtempfindlichkeits-Schwellenwerts in dB gemäß FST mit blauen Lichtreizen. Grau: Daten 1 Monat nach der Behandlung, schwarz: Daten 3 Monate nach der Behandlung. (B) Korrelation zwischen dem Alter und der Verbesserung des dunkeladaptierten Lichtempfindlichkeits-Schwellenwerts in dB, ausgedrückt als durchschnittliche Empfindlichkeitsänderung in der 15-Grad-Makularegion, gemessen mithilfe von DAC-Lichtreizen (cyan). Grau: Daten 1 Monat nach der Behandlung, schwarz: Daten 3 Monate nach der Behandlung. (C) Korrelation zwischen dem Alter und der Verbesserung der Pupillenreaktion auf die skotopischen CPC-Lichtreize, ausgedrückt als durchschnittliche Verbesserung der reIMCA in \% in der 15-Grad-Makularegion. Grau: Daten 1 Monat nach der Behandlung, schwarz: Daten 3 Monate nach der Behandlung. (D) Korrelation zwischen dem Netzhautvolumen, das in vier Quadranten (superior, nasal, inferior, lateral) mit einer Exzentrizität von 3 bis 12 Grad ausgewertet wurde, und der gemittelten relMCA in den entsprechenden Netzhautarealen. Grau: Daten 1 Monat nach der Behandlung, schwarz: Daten 3 Monate nach der Behandlung. (E) Korrelation zwischen dem Netzhautvolumen, das in vier Quadranten (superior, nasal, inferior, lateral) mit einer Exzentrizität von 3 bis $12 \mathrm{Grad}$ ausgewertet wurde, und der Verbesserung der dunkeladaptierten Lichtempfindlichkeit in dB ausgedrückt als durchschnittliche Änderung des Lichtempfindlichkeits-Schwellenwerts in der 15-Grad-Makularegion gemäß DAC (cyan). Grau: Daten 1 Monat nach der Behandlung, schwarz: Daten 3 Monate nach der Behandlung. CPC: chromatic pupil campimetry (chromatische Pupillenkampimetrie); DAC: dark-adapted chromatic perimeter (dunkeladaptierte chromatische Perimetrie); FST: full-field stimulus threshold (Vollfeld-Lichtempfindlichkeits-Schwellenwert-Test); OCT: optical coherence tomography (optische Kohärenztomographie); relMCA: relative maximal constriction amplitude (relative maximale Amplitude der Pupillenverengung).

\section{Prädiktive Faktoren}

Die Zunahme der dunkeladaptierten Lichtempfindlichkeit 1 und 3 Monate nach der Behandlung, gemessen mittels Vollfeld-Methode (FST blau) und anhand des Durchschnitts der DAC-Reize (cyan), korrelierte stark mit dem Alter der Patienten (Abb. 3A, B), wohingegen zwischen dem Anstieg der durchschnittlichen makulären skotopischen CPC-Reaktion und dem Alter nur eine mäBige Korrelation bestand (Abb. 3C).

Darüber hinaus bestand eine mäßige Korrelation zwischen dem Anstieg der Pupillenreaktion in der skotopischen CPC und dem lokalen Netzhautvolumen (ausgewertet in vier Quadranten mit einer Exzentrizität von 3 von 12 Grad, Abb. 3D); zwischen der entsprechenden lokalen Verbesserung der dunkeladaptierten Lichtempfindlichkeit in der DAC (cyan) und der Netzhautdicke bestand dagegen keine Korrelation (Abb. 3E).

\section{Diskussion}

Hauptziel dieser Studie war die Darstellung neuartiger klinischer Protokolle zur individuellen Beurteilung der funktionellen Wiederherstellung der Photorezeptoren in einem klinischen Setting nach Gentherapie. Dazu bewerteten wir das Behandlungsergebnis von fünf Patienten (sieben Augen) bei Baseline sowie 1 Monat und 3 Monate nach der Behandlung mit Voretigen Neparvovec mittels FST, DAC, CPC, BCVA und SD-OCT.

Wir wiesen eine klinisch relevante Verbesserung der dunkeladaptierten Stäbchenempfindlichkeit in einem retinotopen Bereich des behandelten Makula-Areals nach. Ferner zeigen wir erstmals eine objektive retinotop korrekte funktionelle Wiederherstellung, die mittels CPC separat sowohl für die Stäbchen als auch für die Zapfen dokumentiert wurde. Zudem sprechen die Ergebnisse trotz ihrer
160

Kompass Ophthalmol 2021;7:154-163 DOI: $10.1159 / 000519131$ 
Einschränkung durch die geringe Patientenzahl für eine gewisse altersabhängige Verbesserung der dunkeladaptierten Lichtempfindlichkeitsschwelle. Die lokale Netzhautdicke vor der Behandlung war ein besserer Prädiktor für die Verbesserung der Stäbchenfunktion nach der Therapie gemäß skotopischer CPC als das Alter. Über die Wiederherstellung der Zapfen- und Stäbchenfunktion nach einer Gentherapie bei Patienten mit RPE65-Mutation wurde in zahlreichen Veröffentlichungen berichtet, die eine anhaltende Wirkung von bis zu mehreren Jahren zeigten $[9,12,17]$. Die verbesserte Empfindlichkeit der Photorezeptoren gemäß FST während der ersten Wochen betrug in der Regel $<5 \mathrm{~dB}[14,26]$, wobei allerdings auch einige wenige Fälle mit einer FST-Verbesserung von $20 \mathrm{~dB}$ berichtet wurden [16]. In der vorliegenden Arbeit zeigen wir eine Verbesserung von bis zu $45 \mathrm{~dB}$ bei der jüngsten Patientin (14 Jahre). Die Verbesserung der Stäbchenfunktion in unserer Kohorte war vom Alter der Patienten (beim Rückgang des Schwellenwerts) und der verbleibenden Netzhautdicke (beim CPC-Messwert) abhängig. Zwar ist die Zahl der Patienten in unserer Gruppe klein und die Unabhängigkeit der Daten wird durch die Einbeziehung beider Augen teilweise in Frage gestellt, doch deutet der Trend darauf hin, dass das Alter ein wichtiger prädiktiver Faktor ist. Dies kann zusätzlich durch unterschiedliche Therapieeffekte bei Geschwistern (P1/P2 und P3/P4) mit demselben RPE65-Genotyp bestätigt werden. Eine Extrapolation unserer Daten könnte dafür sprechen, dass ein Lebensalter von unter 30 Jahren ein Prädiktor für eine gute Wiederherstellung der Stäbchenfunktion sein könnte. Zudem könnte - unter Berücksichtigung sämtlicher Einschränkungen einer altersbezogenen Analyse - ein lokales Netzhautvolumen von mindestens $450 \mu \mathrm{m}^{3}$ zwischen 3 und 12 Grad retinaler Exzentrizität ein weiterer Prädiktor für die Wirksamkeit der Behandlung sein.

Bei der jüngsten Patientin (P3) erreichte die Verbesserung der Stäbchenfunktion eine dunkeladaptierte Empfindlichkeit von mehr als - $45 \mathrm{~dB}$ und 50\% der normalen Pupillenverengung in der skotopischen CPC. Beide Messwerte (Empfindlichkeit und Verbesserung der Pupillenreaktion) verdeutlichen verschiedene Aspekte der wiederhergestellten Stäbchenfunktion. Kürzlich konnten wir zeigen, dass die Amplitude der Pupillenverengung der Photorezeptordichte (insbesondere beim Zapfensystem) [18, 22] und der Integration in das retinale Netzwerk folgt. Wenn man davon ausgeht, dass die innere Netzhaut bei der jüngsten Patientin (P3) funktionsfähig ist und dass die Intensität der skotopischen CPC-Lichtreize über dem Schwellenwert der reaktivierten Stäbchen liegt (gemäß FST- und DAC-Daten nach der Behandlung), ist zu vermuten, dass die Verbesserung der Pupillenverengung die Dichte der funktionell wiederhergestellten Stäbchen an der untersuchten Netzhautstelle widerspiegelt. Dementsprechend könnte eine Pupillenverengung von 50\% der Norm bei der Patientin ein Hinweis darauf sein, dass etwa 50\% der Stäbchenpopulation des gesunden Auges reaktiviert wurden. Dieser Wert fiel bei den übrigen Patienten geringer aus und lag meist im Bereich von 10 bis 20\%. Ein weiterer Aspekt der CPC-Messungen nach der Behandlung ist die zeitliche Dynamik der Pupillenreaktion. Bei Patientin P3 spricht der Zeitpunkt des Reaktionsbeginns auf sko- topische Lichtreize nach der Behandlung für eine funktionsfähige innere Netzhaut und eine korrekte Integration der Stäbchen in dieses Netzwerk.

Ein wesentliches Ergebnis unserer Analyse war, dass in 3 der 7 Augen nach der Behandlung ein Anstieg der zapfenvermittelten Funktion nachweisbar war. Diese Verbesserung der Retinotopie des behandelten Areals konnte objektiv als Veränderung der CPC-Werte des Zapfenprotokolls nach der Therapie eingestuft werden. Ausgehend von früher veröffentlichten Daten zur Zuverlässigkeit der CPC-Messung [22] geht diese Veränderung bei der jüngsten Patientin (P3) weit über die Variabilität zwischen zwei Messungen hinaus. Bei Patient P8, der die höchste Rate an Stäbchenerholung zeigte, wurde dagegen keine solche Veränderung der lokalen Zapfenfunktion beobachtet. Er wies eine postoperative foveale Vernarbung auf, die negative Auswirkungen auf die subjektive Wahrnehmung des bestkorrigierten Visus hatte und die Verbesserung bei der Beurteilung der Zapfenfunktion mittels CPC verringerte. Zudem war er älter als Patientin P3. Ein stabiler oder nur leicht veränderter bestkorrigierter Visus steht im Einklang mit früheren Veröffentlichungen bei Erwachsenen mit RPE65-Genersatztherapie [16, 17], nach denen die fovealen Zapfen keine einheitliche Verbesserung zeigen. Demgegenüber deuteten einige Studien darauf hin, dass extrafoveale Zapfen auf die Behandlung ansprechen, doch fiel die Interpretation dieses Effekts nicht eindeutig aus $[8,10]$.

Das RPE65-Gen wird im retinalen Pigmentepithel (RPE) exprimiert und ist Bestandteil des Sehzyklus von Stäbchen und Zapfen. Daher wäre die erste Erklärung für die verbesserte Zapfenfunktion, dass die Intervention mit Voretigen Neparvovec die Wiedergewinnung des Chromophors 11-cis Retinal durch RPE65 im RPE auch in den Zapfen direkt beeinflusst. Es ist jedoch bekannt, dass die Wiedergewinnung des Chromophors 11-cis Retinal in den Zapfen nicht nur vom RPE abhängt, sondern dass die Zapfen zudem über einen zweiten Weg über die Müllerzellen verfügen [27]. Verschiedene Veröffentlichungen deuten interessanterweise darauf hin, dass RPE65 nicht nur im RPE, sondern auch in den äußeren Segmenten der Zapfen exprimiert wird [28]. Infolgedessen ist die Veränderung der Zapfenfunktion möglicherweise durch Veränderungen in diesem sekundären Signalweg [27] durch die Reaktivierung der RPE65-Expression zu erklären, wie dies bereits in der Vergangenheit vorgeschlagen wurde [10]. Alternativ könnte die Reaktivierung der Stäbchen auch positive Effekte auf die Zapfenfunktion haben. Es besteht ein Wechselspiel zwischen diesen beiden Systemen über Horizontalzellen, amakrine Zellen und Gap Junctions [29, 30], wodurch es zu einer Modulation der Zapfenfunktion kommen könnte. Technisch betrachtet könnte eine mögliche Erklärung auch sein, dass die lokale Zunahme der Zapfenfunktion lediglich eine Folge der Stäbchen-Reaktivierung an dieser Stelle ist. Die Untersuchung der räumlichen Veränderungen der Zapfenfunktion bei Patientin P3 spricht allerdings dafür, dass die stärkste Veränderung in der Fovea auftritt. Da die Stärke der Zapfenreize vergleichsweise gering ist, ist es sehr unwahrscheinlich, dass die in dieser zentralen Region vernachlässigbare Anzahl an Stäbchen diese Veränderung bewirken könnte. 
Interessanterweise ergab unsere Analyse, dass das Netzhautvolumen vor der Intervention ein Prädiktor für die Verbesserung der CPC-Werte, nicht jedoch der DAC-Werte, nach der Therapie ist. Wie eine kürzlich veröffentlichte Arbeit unserer Gruppe zeigte wiederum mit der Einschränkung eines kleinen Stichprobenumfangs - werden mit der CPC und DAC potentiell unterschiedliche Aspekte der Stäbchenfunktion gemessen [17]. Die Pupillenreaktion auf skotopische CPC-Reize ist, wie oben erörtert, höchstwahrscheinlich eine Funktion der Anzahl an Stäbchen. Da die Netzhautdicke bis zu einem gewissen Ausmaß ein Indikator für die morphologisch vorhandenen Photorezeptoren ist [31], könnte die Veränderung der Stäbchen-Reaktion in der CPC die Anzahl der reaktivierten Stäbchen an der untersuchten Stelle widerspiegeln. Dagegen handelt es sich beim DAC-Reiz um einen Schwellenwert-Stimulationstyp, der vermutlich nicht so sehr von der Anzahl der verfügbaren Zellen abhängt, sondern von ihrer Empfindlichkeit, die von der Länge der äußeren Segmente abhängig sein kann. Dies könnte eine Erklärung für die fehlende Korrelation zwischen dem Netzhautvolumen vor der Operation und der DAC-Verbesserung sein.

Darüber hinaus ist eine hohe Korrelation zwischen dem Lebensalter und der Verbesserung der dunkeladaptierten Empfindlichkeit nach der Behandlung in der FST (blau) und DAC (cyan) zu beobachten. Zwar ist das Alter möglicherweise ein Prädiktor für die Anzahl der verbleibenden lebensfähigen Stäbchen, was die moderate Korrelation mit der Verbesserung in der skotopischen CPC erklären könnte (Abb. 3C), allerdings scheint es ein validerer Prädiktor für das Behandlungsergebnis bei der dunkeladaptierten Empfindlichkeit zu sein (Abb. 3A, B). Dies deutet darauf hin, dass die Chancen für eine Wiederherstellung der Stäbchenfunktion mit zunehmendem Alter sinken.

Unsere Untersuchung weist verschiedene Einschränkungen auf. Eine Einschränkung besteht in der geringen Patientenzahl, die durch die Seltenheit von RPE65-Mutationen und die Verfügbarkeit von Patienten für diese Behandlung bedingt ist. Dies hat insbesondere Auswirkungen auf die Unabhängigkeit der Daten, da bei einigen Patienten beide Augen und mehrere Regionen desselben Auges in die Korrelationsanalyse eingeschlossen wurden. Der therapeutische Effekt war jedoch für beide Augen unabhängig und wurde für beide Augen zu unterschiedlichen Zeitpunkten erfasst. Wegen der schlechten Fixation einiger Patienten konnten zudem nicht bei allen Patienten OCT-Scans des Netzhautvolumens erstellt werden. Daher sind unsere Ergebnisse mit Vorsicht $\mathrm{zu}$ interpretieren.

Eine weitere Einschränkung unserer Arbeit könnte die kurze Beobachtungszeit von bis zu 3 Monaten sein. Weitere Untersuchungen mit Längsschnittdatenanalysen mit den hier vorgeschlagenen Methoden sind erforderlich, um das Potenzial der retinotopischen Wiederherstellung von Stäbchen und Zapfen nach einer Gentherapie zu beurteilen.

Mit den hier vorgestellten Messungen der Netzhautfunktion lassen sich die subjektiven und objektiven Ergebnisse der Zapfenund Stäbchenfunktion mit bislang nicht gekannter räumlicher Auflösung ermitteln. Da Patienten mit erblicher Netzhautdege- neration häufig heterogene Phänotypen aufweisen, kann das individuelle Degenerationsmuster mithilfe dieser Tests leicht überwacht werden. Die hier beschriebenen Methoden können Erkenntnisse zu verschiedenen Aspekten des Behandlungseffekts liefern, von der Wiederherstellung der Zellpopulation (CPC) bis zur Verbesserung der Empfindlichkeit im dunkeladaptierten $\mathrm{Zu}$ stand (DAC, FST). Diese Methoden könnten ein neues Kapitel in der Bewertung der Gentherapieeffekte aufschlagen.

\section{Fußnoten}

M. D. F. und K. S. haben zu gleichen Teilen zur Erstellung dieser Arbeit beigetragen.

\section{Beitragende Autoren}

Kr. S. trug zum Studiendesign, zur Datenanalyse und zur Erstellung des Manuskriptentwurfs bei. Ka. S. trug zum Studiendesign, zur Datenerhebung und zur Erstellung des Manuskriptentwurfs bei. D. F. trug zur Konzeption der Studie, zur Datenerhebung und zur Überarbeitung des Manuskripts bei. M. K., R. J., F. R., F. W., F. C. K., F. N., S. D., E. Z., Be. W., S. K. und K. U. B.-S. trugen zur Datenerhebung und zur Überarbeitung des Manuskripts bei. Ba. W., T. P. und C. K. trugen zum Design eines Studienteils und zur Überarbeitung des Manuskripts bei. Ka. S. und D. F. waren zu gleichen Teilen an der Erstellung dieses Manuskripts beteiligt. Alle Autoren genehmigten die finale Fassung des Manuskripts und erklären ihr Einverständnis, Verantwortung für alle Aspekte der Arbeit zu übernehmen.

\section{Finanzierung}

Die vorliegende Arbeit wurde durch die Deutsche Forschungsgemeinschaft (Förderkennzeichen: STI 727/1-1 und 276693517), die Tistou and Charlotte Kerstan Stiftung (Förderkennzeichen: RD-CURE WP4/5-1) sowie die Egon Schumacher-Stiftung, Barnstorf, eine private Stiftung ohne kommerzielle Interessen finanziell unterstützt. D. F. erhielt finanzielle Unterstützung durch die Deutsche Forschungsgemeinschaft (Förderkennzeichen: FI 2336/1-1), das Deutsche Förderprogramm für Augenheilkunde, die Henning Zügel Stiftung, die Hermann Wacker Stiftung und ProRetina e.V.

\section{Interessenkonflikte}

D. F. ist oder war Beiratsmitglied und/oder Berater und/oder erhielt Honorare/Forschungsgelder/Reisekostenzuschüsse von folgenden Unternehmen: Adelphi Values, Advent France Biotechnology, Alphasights, Atheneum, Axiom Healthcare Strategies, Biogen, Decision Resources, Dialectica, EyeServ, Frontera Therapeutics, Janssen Research \& Development, Navigant, Novartis, Roche, RegenxBio, Sirion und STZ eyetrial. D. F. ist Direktor von Fischer Consulting Limited und hält ein Patent (50\%) an einem Gentherapieprodukt zur Behandlung der X-chromosomalen Retinitis pigmentosa. D. F. ist der leitende Prüfer der globalen (außer USA) multizentrischen, multinationalen, longitudinalen, Beobachtungs-Post-Authorisation-Safety-Studie bei Patienten, die mit Voretigen Neparvovec (CLTW888A12401) behandelt wurden und die hier vorgestellten Daten sind zum Teil in dieser Studie enthalten. E. Z. ist oder war Beiratsmitglied und/ oder Berater und/oder erhielt Honorare/Forschungsgelder/Reisekostenzuschüsse von folgenden Unternehmen: SMERUD, Atheneum, Axiom Healthcare strategies, Biogen, Decision Resources, Janssen Research \& Development, Navigant, RegenxBio, ProQR, Gyroscope, Astellas und STZ eyetrial. Ka. S. erhielt Forschungsgelder und/oder Reisekostenerstattungen von Novartis, ProQR und ORA. S. K. und F. W. erhielten persönliche Honorare von Novartis. Ba. W. und T. P. erhielten anderweitige Unterstützung von Novartis.

\section{Genehmigung durch die Ethikkommission}

Ethikkommission der Medizinischen Fakultät der Universität Tübingen (328/2020BO2). 


\section{Erklärung zur Datenverfügbarkeit}

Alle für die Studie relevanten Daten sind im Artikel enthalten oder als ergänzende Informationen hochgeladen. Das Manuskript enthält die pseudonymisierten Daten der Patienten, die in dieser Analyse ausgewertet wurden.

\section{Lizenzangabe}

Stingl K, Kempf M, Bartz-Schmidt KU, Dimopoulos S, Reichel F, Jung R, Kelbsch C, Kohl S, Kortüm FC, Nasser F, Peters T, Wilhelm B, Wissinger B, Wozar F, Zrenner E, Fischer MD, Stingl K. Spatial and temporal resolu- tion of the photoreceptors rescue dynamics after treatment with voretigene neparvovec. Br J Ophthalmol. 2021 Jan 20:bjophthalmol-2020-318286. (DOI: 10.1136/bjophthalmol-2020-318286) O Author(s) (or their employer(s)) 2021 (Übersetzung; Zustimmung der Patienten zur Veröffentlichung und Provenienz, Peer Review, Open access und ORCID iDs gekürzt), lizensiert unter CC BY 4.0 (https://creativecommons.org/licenses/by/4.0/deed.de).

\section{Literatur}

Die Literatur ist unter www.karger.com/doi/10.1159/000519131 abrufbar. 